\title{
Differential Regulation of Salmonella Typhimurium Genes Involved in O-Antigen Capsule Production and Their Role in Persistence Within Tomato Fruit
}

\author{
Massimiliano Marvasi, ${ }^{1}$ Clayton E. Cox, ${ }^{1}$ Yimin $\mathrm{Xu},{ }^{2}$ Jason T. Noel, ${ }^{1}$ James J. Giovannoni, ${ }^{2}$ and \\ Max Teplitski ${ }^{1}$ \\ ${ }^{1}$ Soil and Water Science Department, Genetics Institute Rm330E, 2033 Mowry Rd, University of Florida-IFAS, Gainesville \\ 32611, U.S.A.; ${ }^{2}$ United States Department of Agriculture-Agricultural Research Service and Boyce Thompson Institute for \\ Plant Research, Tower Road, Cornell University, Ithaca, NY 14853, U.S.A.
}

Submitted 4 September 2012. Accepted 6 March 2013.

\begin{abstract}
Enteric pathogens, including non-typhoidal Salmonella spp. and enterovirulent Escherichia coli, are capable of persisting and multiplying within plants. Yet, little is still known about the mechanisms of these interactions. This study identified the Salmonella yihT gene (involved in synthesis of the $O$-antigen capsule) as contributing to persistence in immature tomato fruit. Deletion of yihT reduced competitive fitness of $S$. enterica sv. Typhimurium in green (but not ripe, regardless of color) tomato fruit by approximately 3 logs. The yihT recombinase-based in vivo expression technology (RIVET) reporter was strongly activated in unripe tomato fruit, and fitness of the mutant inversely correlated with the level of the yihT gene expression. Expression of yihT in mature tomato fruit was low, and yihT did not affect competitive fitness within mature fruit. To better understand the molecular basis of the phenotype, behaviors of the yihT RIVET reporter and the yihT mutant were tested in tomato fruit defective in ethylene signaling. These experiments suggest a role for functional ethylene-mediated signaling in the persistence of Salmonella spp. within tomato fruit. Furthermore, jasmonic acid and its precursors strongly reduced expression of yihT.
\end{abstract}

Over the past decade, at least a dozen major outbreaks of salmonellosis have been traced to the consumption of fresh fruit and vegetables (Batz et al. 2011; Mandrell 2009). By some estimates, the burden of the non-typhoidal Salmonella infections contracted as a result of raw produce consumption is comparable with the burden of salmonellosis caused by foods of animal origin (Batz et al. 2011). In the aftermath (and, likely, as a consequence) of each produce-linked outbreak of gastroenteritis, the commodity price drops dramatically (Teplitski et al. 2012). Initially, it was hypothesized that these outbreaks are a result of poor sanitation during various production stages. However, produce-linked outbreaks still occur even after significant science-driven improvements in pre- and postharvest handling of fresh produce. It is now well accepted that, under suitable conditions, enteric pathogens are capable of colo-

Corresponding author: M. Teplitski; E-mail: maxtep@ufl.edu

* The $e$-Xtra logo stands for "electronic extra" and indicates that one supplementary table is published online.

(C) 2013 The American Phytopathological Society nizing and persisting within various plant tissues in the field (Danyluk et al. 2008; Greene et al. 2008; Uesugi et al. 2007), possibly as a part of their normal lifecycle (Brandl et al. 2013). Furthermore, growth within plants quickly selects for variants and spontaneous mutants of both Salmonella enterica and enterovirulent Escherichia coli that are more fit for habitation in plant tissues (Parker et al. 2012; Zaragoza et al. 2012). Despite the apparent importance of the plant-associated lifestyle in the ecology of enterics, relatively little is still known about mechanisms of interactions between enterics and plants, and even less is known about the genetics of Salmonella-tomato interactions.

Colonization of tomato surfaces and vegetative and reproductive tissues depends on specific Salmonella genes and is a function of the tomato genotype and the physiological state of the plant and specific tissues (Barak and Schroeder 2012; Barak et al. 2011; Noel et al. 2010a; Shi et al. 2007). Approximately 2-log differences were observed in the ability of the same Salmonella strain to colonize surfaces of tomato fruit of different species and cultivars (Barak et al. 2011), highlighting the importance of plant genotype in the outcome of plant interactions with enterics. Salmonella genetics also contributes to the persistence of the pathogen in plants in general (Tyler and Triplett 2008) and in tomato in particular (Barak and Schroeder 2012; Shi et al. 2007). Salmonella spp. horizontally acquired virulence genes do not appear to play a role in persistence of this pathogen within mature tomato fruit (Noel et al. 2010a), suggesting that Salmonella spp. use an entirely different set of genes for their interactions with plants (Teplitski et al. 2012).

A screen of $S$. enterica serovar Typhimurium promoters for those differentially regulated within tomato identified approximately 50 unique Salmonella genes that were up- or downregulated within red ripe tomato fruit compared with in vitro growth (Noel et al. 2010a). However, none of the corresponding mutations in individual Salmonella genes significantly reduced the pathogen's competitive fitness within red ripe tomato fruit. A significant reduction in persistence was only observed after mutations in five of these genes were combined in the same Salmonella strain (Noel et al. 2010a), thus suggesting that multiple Salmonella sensory, regulatory, and structural inputs contribute to the outcome of the interactions of this bacterium with plants. The rdar wild-type phenotype (observed as red, dry wrinkled colonies on low-salt plates containing the Congo Red dye) (Gibson et al. 2006; Romling et al. 2000) strongly correlates with the fitness of Salmonella spp. in many 
plant interactions (Barak and Schroeder 2012; Brandl et al. 2013; Zaragoza et al. 2012). However, it is not yet certain whether it was the multicellular rdar phenotype itself or the regulatory contribution of $\mathrm{AfgD}$, which is one of the many regulators of $r d a r$ (Gibson et al. 2006; Romling et al. 2000), that was responsible for the increased fitness of Salmonella spp. within plants. To address this uncertainty, this study focused on the role of the AgfD-controlled yih genes involved in O-antigen capsule synthesis and their role in the persistence of Salmonella spp. within tomato fruit.

The Salmonella O-antigen capsule is encoded by the divergent operons yihU-yshA and yihV-yihW (Gibson et al. 2006). These two operons are differently controlled by AgfD, a key regulator of multicellular behavior in Salmonella spp., even though they do not contribute to the rdar phenotype (Gibson et al. 2006; Romling et al. 2000). None of the genes encoded within the cluster STM4010-STM4030, which includes the $y i h U-y s h A$ and $y i h V-y i h W$ operons, has a consistent fitness defect in the spleen, lung, or liver tissues of mice (even though the mutants are apparently viable under common laboratory conditions because they were present within the tested libraries) (Arrach et al. 2010). Similarly, a screen of 1,045 signaturetagged mutants of serovar Typhimurium in chicks or calves did not identify any of the genes within this cluster (Morgan et al. 2004), although it is not known whether these genes were present in the tested library. A mutation in yihT did not affect fitness of serovar Typhimurium in mice infected with the pathogen intraperitoneally (other genes from the yih operons were not in the tested libraries) (Santiviago et al. 2009). Col- lectively, these results suggest that the yihU-yshA, yihV-yihW genes are either not involved in virulence or are not expressed inside warm-blooded animals. Consistent with this hypothesis, the $y i h U-y s h A$ putative operon of serovar Enteritidis was not expressed in Luria-Bertani medium (LB) or in human macrophage cells, whereas the divergently encoded yihVW genes were co-regulated and expressed at low basal levels in LB and in human macrophages. The predicted yihX-yihZ operon was strongly expressed in LB and in macrophages (Ge et al. 2010). Furthermore, none of the promoters of these operons in serovar Typhimurium was identified as differentially regulated in promoter probe screens of genes activated inside mouse tissues (Arrach et al. 2008). Interestingly, the yihO gene was identified in a screen of Salmonella mutants unable to attach to alfalfa sprouts (Barak et al. 2007). Collectively, these observations suggest that the $\mathrm{O}$-antigen capsule encoded by the $y i h U$ $y s h A, y i h V-y i h W$ operons functions in ecological niches outside of warm-blooded hosts, possibly including plants.

This study revealed that $y i h T$ is differentially regulated in red and green tomato fruit even though no phenotype for the O-antigen capsule mutants was observed in a previous study with red ripe tomato fruit (Noel et al. 2010a). Deletion of the yihT gene strongly reduced fitness of Salmonella spp. in green tomato fruit as well as in tomato mutants with disrupted ethylene synthesis and signaling. The results offer an important connection between the functions of specific Salmonella genes and persistence within plant tissues, and specifically point to plant ethylene-mediated defense and ripening-related pathways as possible candidates for plant processes that facilitate hosting

\section{Fitness of Salmonella $\Delta y i h T$ in tomatoes}

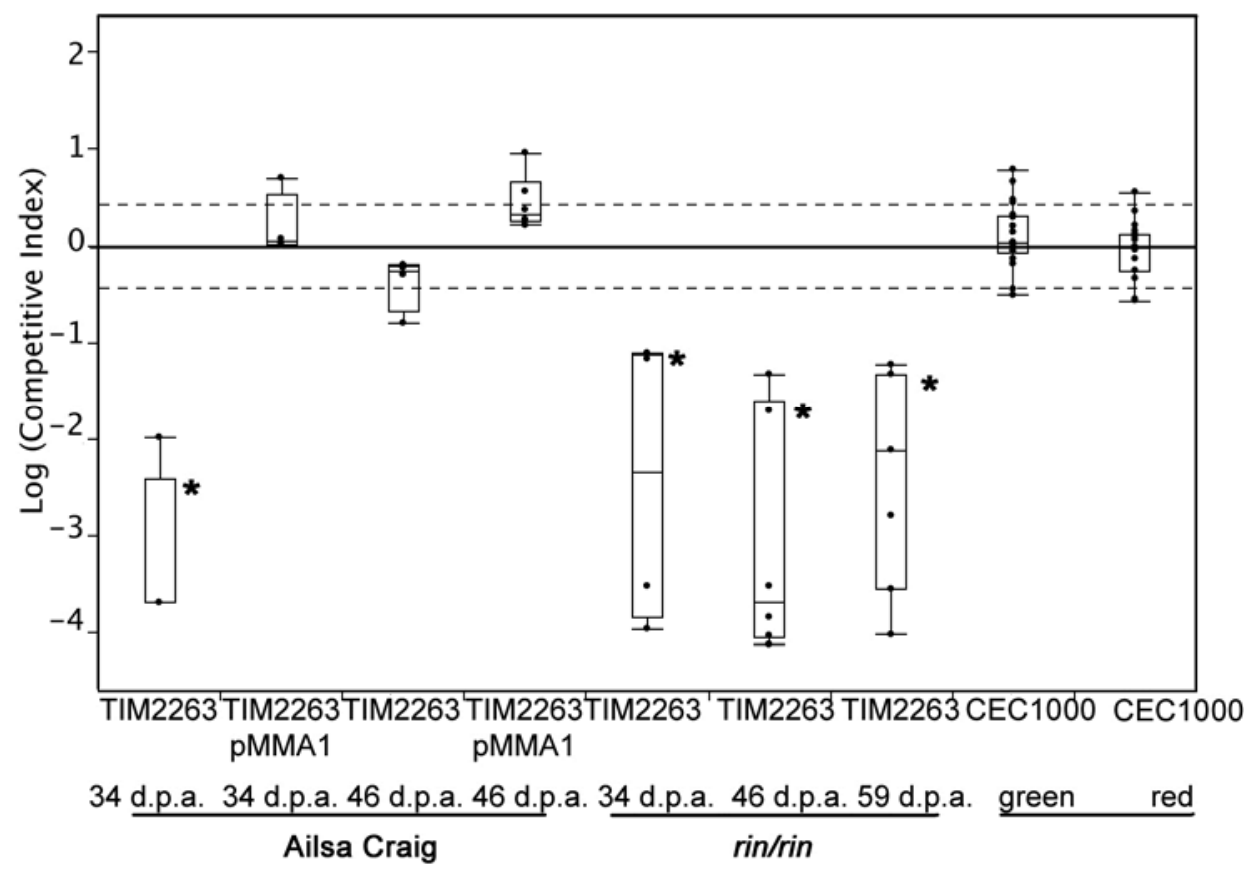

Fig. 1. Competitive fitness of the yihT mutant in red and green tomato fruit of 'Ailsa Craig' and rin/rin mutant. The yihT mutant Salmonella enterica sv. Typhimurium TIM2263 (marked with the kanamycin resistance cassette) or complemented strain TIM2263 pMMA1 were co-infected in a 1:1 ratio with the isogenic wild-type serovar Typhimurium 14028 into shallow wounds made in the tomato epidermis with a sterile paper clip. The inoculum dose was estimated by dilution plating to be approximately 50 to $100 \mathrm{CFU} /$ infection. Tomato fruit were incubated for 7 days at $22^{\circ} \mathrm{C}$ and $41 \%$ relative humidity prior to maceration (stomaching) in phosphate-buffered saline and plating onto xylose-lysine deoxycholate medium. Ratios of the wild type to the mutant in the inoculum and in the samples recovered from tomato were estimated by patching on antibiotic-containing plates. Experiments were conducted with at least four biological replicates. Statistical significance was determined by a $t$ test against similarly conducted co-infections of serovar Typhimurium 14028 and serovar Typhimurium CEC1000 (marked with a kanamycin-resistance cassette in a presumed neutral site of the genome). Tomato fruit were harvested at the indicated time points $(34,46$, or 59 days postanthesis [d.p.a.]) to synchronize developmental stages in tomato fruit, as indicated under the figure. Experiments were conducted in 'Ailsa Craig' or its nearly isogenic rin/rin mutant, as indicated in the figure. In box plots, boxes include the lower and upper quartiles, lines within the box are the medians, and whiskers indicate the degree of dispersion of the data. Dashed lines estimate a cut-off beyond which changes in competitive fitness are biologically and statistically significant. 
Salmonella spp. and, possibly, other pathogenic or nonpathogenic bacteria.

\section{RESULTS AND DISCUSSION}

\section{Deletion of yihT strongly reduces fitness}

in immature tomato fruit.

In our previous experiments with red ripe fruit of 'Campari' tomato, a deletion of yihT or the entire yihT-ompL cluster did not affect fitness of the mutants (Noel et al. 2010a), even though yihT was expressed within tomato fruit (Zaragoza et al. 2012). Consistent with these earlier studies, a deletion of yihT did not strongly reduce competitive fitness of the mutant in red (46 days postanthesis [dpa]) 'Ailsa Craig' tomato (Fig. 1). However, competitive fitness of the mutant (measured in the presence of the isogenic wild type strain) in mature green (34 dpa) tomato fruit harvested from the same plant was reduced by 100 - to 1,000 -fold (Fig. 1). The mutation was fully complemented with a copy of the yihUT gene cluster (driven by the

Table 1. Competitive fitness of the $\Delta y i h T$ in tomato fruit exposed to ethylene

\begin{tabular}{llc}
\hline & \multicolumn{2}{c}{ Mean of the log competitive index $\pm \mathbf{S E}^{\mathbf{a}}$} \\
\cline { 2 - 3 } Genotype (dpa) $^{\mathbf{b}}$ & No treatment & Ethylene treatment \\
\hline 'Ailsa Craig' (34, green) & $-3.272 \pm 0.32$ & $-0.465 \pm 0.26$ \\
'Ailsa Craig' (46, red) & $-0.382 \pm 0.32$ & $0.600 \pm 0.37$ \\
rin (34) & $-2.445 \pm 0.32$ & $-1.142 \pm 0.32$ \\
rin (46) & $-3.098 \pm 0.26$ & $-0.440 \pm 0.26$ \\
\hline
\end{tabular}

${ }^{\mathrm{a}} \mathrm{SE}=$ standard error. Statistically significant effects $(P<0.05)$ of ethylene treatment are shown in bold.

${ }^{\mathrm{b}}$ Tomato genotype at days postanthesis (dpa). native and lac promoters) on a low-copy-number vector (Fig. 1). This suggests that the physiological or maturation state of the tissue dramatically affects persistence of Salmonella spp. within the fruit. Aside from the implications in food safety, these observations indicate that either Salmonella spp. have evolved mechanisms to gauge the suitability of alternate hosts (such as plants) for infection or their plant hosts differentially inhibit endophytic bacterial colonists depending on their developmental or physiological state and in response to the detection of the specific bacterial surface antigens. Therefore, the following experiments focused on defining the behavior of the yihT mutant in tomato fruit defective in ripening or pigment production.

\section{Fitness of Salmonella $\mathrm{O}$-antigen capsule mutant} in tomato fruit defective in ethylene perception.

In tomato, ethylene-mediated signaling contributes to ripening as well plant defenses. Therefore, the role of the tomato ethylene pathway in the fitness of the Salmonella O-antigen capsule mutant was tested. In the tomato ripening-inhibitor (rin) mutant, a SEPALATA MADS-box gene encoding a necessary regulator of fruit ripening is disrupted. LeMADS-RIN, a global regulator of ripening, is required to initiate ethylene biosynthesis in addition to ripening factors that cannot be complemented by supplemental ethylene (Martel et al. 2011; Vrebalov et al. 2002). When the Salmonella yihT mutant was tested in the tomato rin/rin mutant, its fitness was strongly reduced, similarly to its reduced fitness in the green fruit of the nearly isogenic tomato 'Ailsa Craig' (Fig. 1).

The treatment of fruit of 'Ailsa Craig' and rin/rin mutants with ethylene alleviated the fitness defect of the Salmonella yihT mutant (Table 1) in immature (34 dpa) fruit of 'Ailsa Craig' and

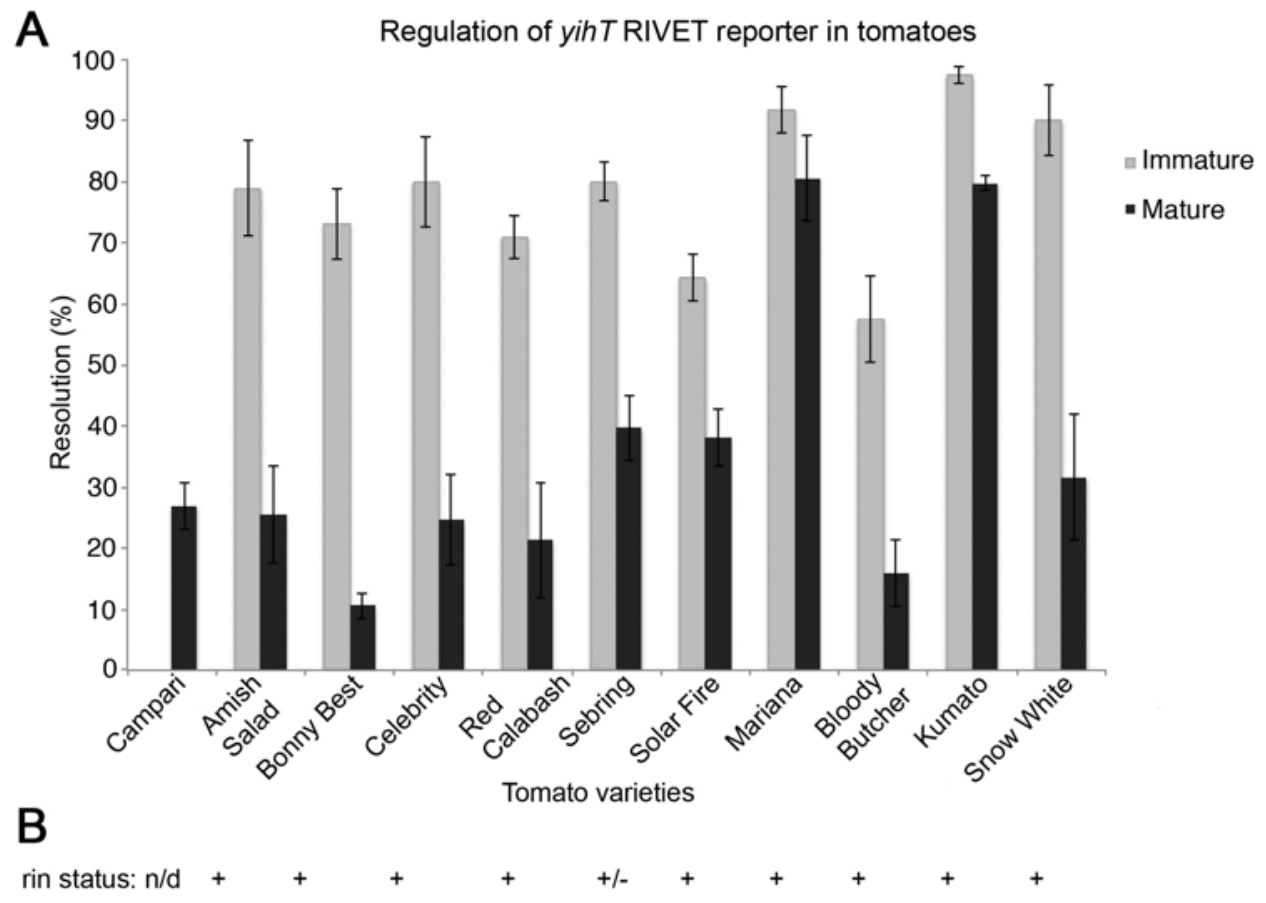

Fig. 2. Resolution of the $y i h T$ recombinase-based in vivo expression technology (RIVET) reporter in red and green tomato fruit of different commercial varieties. A, Cultures of JTN203 were grown overnight from glycerol stock in Luria-Bertani (LB) broth containing tetracycline and washed three times in sterile, deionized water; then, approximately $150 \mathrm{CFU} /$ infection were inoculated into shallow wounds in the tomato epidermis. Following a week-long incubation, each infection was sampled, and colonies formed on xylose-lysine deoxycholate with kanamycin were patched on LB containing tetracycline to determine the resolution of the reporter. 'Campari' tomato fruit (red, unwaxed, sold in a "clam"-type plastic container) were purchased from local supermarkets. All other tomato fruit were grown either under field conditions (conventional in fall 2010 and 2012 in Lake City, FL or transitional organic in spring 2011 and 2012 in Archer, FL) or in a roof-top greenhouse on the University of Florida campus. The yihT RIVET reporter was tested in both field- and greenhousegrown tomato fruit of each variety except for Campari. B, Status of the rin allele in the tested tomato varieties is shown. 'Sebring' is heterozygous for rin, while all other cultivars are homozygous for the wild-type Rin allele. 
in the rin/rin fruit (34 and $46 \mathrm{dpa}$ ), and had no effect in mature (46 dpa) fruit of 'Ailsa Craig'. Even though the ethylene-dependent rescue of the fitness of the yihT mutant was statistically significant, fitness was not completely restored to the level of the wild type. Because ripening in the rin/rin mutant cannot be restored by ethylene, the ability of the exogenously added ethylene to at least partially restore fitness of the Salmonella yihT mutant suggests that both LeMADS-RIN and ethylene affect the ability of Salmonella spp. to persist in tomato fruit.

\section{Regulation of the Salmonella $\mathrm{O}$-antigen capsule genes in tomato ripening and ethylene mutants.}

To better understand how Salmonella O-antigen capsule genes contribute to the ability of this human pathogen to interact with plants, regulation of the $y i h T$ and $y i h X$ recombinasebased in vivo expression technology (RIVET) reporters was tested in mature and immature fruit of tomato 'Ailsa Craig' and its isogenic rin/rin and $\mathrm{Nr} / \mathrm{Nr}$ mutants at 34 (mature green), 46 (ripe), and 59 dpa. The $\mathrm{Nr}$ locus encodes an ethylene receptor with impaired ethylene-binding capacity causing delayed and incomplete ripening of the $\mathrm{Nr}$ mutants due to reduced ethylene sensitivity (Yen et al. 1995). The $\mathrm{rin} / \mathrm{rin}$ and $\mathrm{Nr} / \mathrm{Nr}$ fruit were the same dpa as the wild-type 'Ailsa Craig' control but mature to green and orange, respectively, by 59 days.

In vivo reporters (such as RIVET) have an important advantage for documenting Salmonella gene expression within alternate hosts (such as plants) (Teplitski et al. 2012). RIVET is based on the activation of a recombinase (typically, TnpR) cloned downstream of the promoter of interest. TnpR then excises a selectable marker (resistance to tetracycline, for example), and the loss of the marker is then scored quantitatively by patching colonies of bacteria recovered from the host onto plates containing antibiotics (Angelichio and Camilli 2002). RIVET works well in Salmonella spp. and is as sensitive as the lac $Z$ reporter in vitro; however, there are important caveats in the interpretation of the RIVET data in general (Merighi et al. 2005; Noel et al. 2010b) and as it relates to the Salmonella spp.-plant interactions (Teplitski et al. 2012). The measurable resolution of the yihT RIVET reporter in soft LB agar is negligible and is increased when the reporter is incubated inside tomato fruit (Zaragoza et al. 2012).

Expression of the yihT RIVET reporter in $\mathrm{rin} / \mathrm{rin}$ tomato at all three maturity stages was high, and indistinguishable from the activity of the reporter in immature (34 dpa) fruit of the parental 'Ailsa Craig' (Fig. 2A). In the $\mathrm{Nr} / \mathrm{Nr}$ tomato (reduced response to ethylene), resolution of the $y i h T$ RIVET reporter was high at 46 days (comparable with mature green, 34 dpa tomato fruit of the parent). In 59-dpa $\mathrm{Nr} / \mathrm{Nr}$ tomato fruit, the resolution of the reporter decreased to the levels comparable with the red ripe (46 days old) wild type. Because these tomato mutants lack ripening ethylene synthesis (rin) or are deficient in their ability to respond to it $(\mathrm{Nr})$, these observations suggest that the upregulation of yihT inside green tomato fruit (or downregulation in red tomato fruit) is a consequence of fruit maturity indicators, possibly including ethylene-dependent changes in tomato defense responses or physiology.

Table 2. Contribution of tomato metabolites to the expression of yihT in Salmonella enterica JTN203

\begin{tabular}{lcc}
\hline & \multicolumn{2}{c}{ Mean $\boldsymbol{y i h T}$ resolution $(\boldsymbol{\%})( \pm \mathbf{S E})^{\mathbf{a}}$} \\
\cline { 2 - 3 } Carbon sources $^{\mathbf{b}}$ & M9 & $\mathbf{1 / 1 0 ~ L B ~}$ \\
\hline $\mathrm{H}_{2} \mathrm{O}$ (control) & $26 \pm 4$ & $5 \pm 2$ \\
Organic, green $^{\mathrm{c}}$ & $16 \pm 2$ & $5 \pm 2$ \\
Organic, red $^{\mathrm{d}}$ & $9 \pm 4$ & $2 \pm 1$ \\
Glucose $\Uparrow_{\text {Sucrose } \Downarrow}$ & $14 \pm 3$ & $8 \pm 3$ \\
Glycerol & $31 \pm 10$ & $3 \pm 1$ \\
Mannitol $\Downarrow$ & $6 \pm 4$ & $8 \pm 3$ \\
Myo-inositol $\Downarrow$ & $16 \pm 4$ & $8 \pm 3$ \\
Arginine $\Downarrow$ & $16 \pm 6$ & $4 \pm 1$ \\
Glycine $\Downarrow$ & $17 \pm 6$ & $5 \pm 2$ \\
Linoleic acid $\Downarrow$ & $25 \pm 4$ & $9 \pm 4$ \\
Palmitic acid $\Downarrow$ & $1 \pm 0.5$ & $1 \pm 0.4$ \\
$\gamma$-linolenic acid & $26 \pm 0.5$ & $5 \pm 1$ \\
13(S)-HpOTrE $(\gamma)$ & $2 \pm 1$ & $2 \pm 1$ \\
12-oxo-phytodienoic acid & $16 \pm 3$ & $5 \pm 2$ \\
Jasmonic acid & $10 \pm 2$ & $2 \pm 1$ \\
Decanoic acid (Control) & $2 \pm 1$ & $1 \pm 0.4$ \\
\hline
\end{tabular}

${ }^{a}$ Mean yihT reporter resolution \pm standard error $(\mathrm{SE})$ and $\mathrm{LB}=$ LuriaBertani medium.

${ }^{b}$ An upward arrow indicates an increase in accumulation of the metabolite in tomato fruit as they mature (Carrari et al. 2006) and a downward arrow indicates that accumulation of the metabolite decreases as the fruit matures. Glycerol is present at the same amount in red and green tomato fruit (Carrari et al. 2006).

c Organic extract, 'Ailsa Craig' green tomato, 34 days postanthesis (dpa).

d Organic extract, 'Ailsa Craig' red tomato, $46 \mathrm{dpa}$.
A

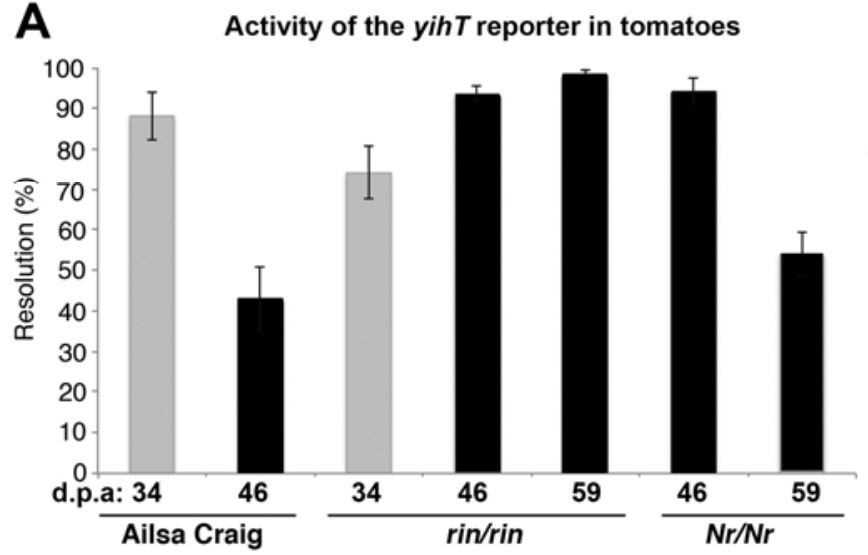

B Activity of the yihX reporter

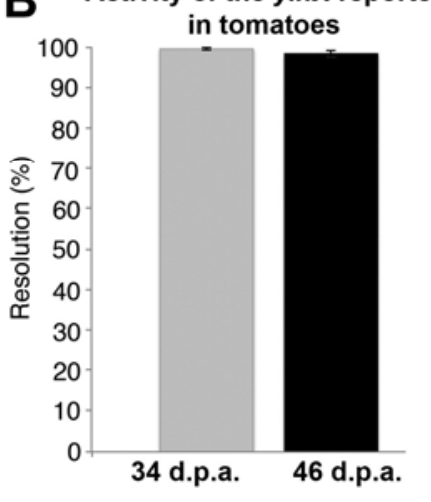

Fig. 3. Resolution of the yih recombinase-based in vivo expression technology (RIVET) reporters in tomato 'Ailsa Craig'. A, Resolution of the yihT RIVET reporter was tested in red ripe (46 days postanthesis [d.p.a.]) and mature green (34 d.p.a.) fruit of 'Ailsa Craig'. Resolution of the reporter was similarly tested in isogenic tomato mutants with defects in ethylene production ( $\mathrm{rin} / \mathrm{rin}$ ) or impaired response to ethylene ( $\mathrm{Nr} / \mathrm{Nr}$ ). Because ripening of these mutants is inhibited, neither physiological nor visual assessment of ripening is applicable. Thus, sampling was synchronized to the wild type by tagging tomato fruit and harvesting at the same age as the wild type. Four to six biological replications were carried out and averages of all experiments are shown; error bars are standard deviations. B, Resolution of the yihX RIVET reporter in green (34 d.p.a.) and red (46 d.p.a.). As a control, the reporter was tested in soft LuriaBertani agar, and the resolution was found to be $87 \pm 3 \%$. 
As a control, activity of the $y$ ihX RIVET reporter was tested under the same conditions. The yihX RIVET reporter was strongly expressed in LB $(87 \pm 3 \%)$, consistent with microarray data in serovar Enteritidis (Ge et al. 2010). The yihX reporter was expressed in mature and immature tomato of 'Ailsa Craig' (Fig. 3). This suggests that the responses of the yihT RIVET reporter observed in this study are specific to this operon and are not artifactual.

\section{Expression of the yihT RIVET reporter in commercial tomato varieties.}

To follow up on the experiments with the fitness of the Salmonella yihT O-antigen capsule mutant in tomato defective in ethylene synthesis and perception, yihT-dependent phenotypes were tested in commercial tomato varieties.

The yihT RIVET reporter was resolved at 10 to $40 \%$ in red ripe tomato fruit of 'Campari', 'Amish Salad', 'Bonny Best', 'Celebrity', 'Red Calabash', 'Sebring', and 'Bloody Butcher' (Fig. 2). A higher level of resolution (approximately 80\%) was observed in ripe tomato fruit of 'Mariana' and 'Kumato'. A much higher, and fairly uniform, level of resolution (60 to $100 \%$ ) was observed in green tomato fruit of all varieties. The presence of pigments per se does not seem to affect the activity of the reporter, because the fruit of Mariana turn red when mature whereas mature fruit of Kumato appear brown (because, in addition to the red pigments, they retain chlorophyll due to the green-flesh $(g f)$ mutation in a STAY-GREEN protein coding gene) (Barry et al. 2008). However, resolution of the reporter in mature tomato fruit of both of these varieties seems higher than in other tomato fruit that turn red when mature. Therefore, it is reasonable to hypothesize that major pigments associated with ripening did not affect the resolution of the yihT RIVET reporter.

Because some commercial tomato varieties are heterozygous for rin, tomato varieties in which expression of the Salmonella yihT was tested were genotyped. Of the 10 tested varieties, only 'Sebring' was heterozygous for rin. Even though expression of Salmonella yihT inside red tomato fruit of 'Sebring' was higher than in 'Campari', 'Amish Salad', 'Bonny Best', 'Celebrity', 'Red Calabash', and 'Bloody Butcher', it was the same as in 'Solar Fire' and 'Snow White' and less than in 'Mariana' and 'Kumato' (all four of which carry the wildtype allele of Rin) (Fig. 3).

Differences in the resolution of the yihT reporter in tomato fruit of 'Kumato', 'Snow White', and 'Mariana' (Fig. 2) prompted us to investigate whether fitness of the mutants in ripe fruit of these varieties is inversely proportional with the level of the yihT expression, as has been observed in green fruit and in rin tomato fruit (Figs. 1 and 3). The competitive fitness of the yihT mutant in ripe tomato fruit of 'Kumato', 'Snow White', and 'Mariana' was $-0.75 \pm 0.16,1.24 \pm 0.23$, and $0.93 \pm 0.13$, respectively. Even though fitness of the mutant in 'Kumato' tomato was reduced, this reduction was not statistically significant (when compared with co-infections of the wild type and a neutral kanamycin-resistant mutant). In tomato fruit of 'Snow White' and 'Mariana', the mutant was modestly but statistically significantly more fit than the wild type. Thus, the inverse correlation between the level of the yihT expression and the fitness of the mutant is consistent in unripe tomato fruit and in the tomato mutants defective in ripening and ethylene signaling. What cues are responsible for the differential regulation of $y i h T$ in green versus ripe tomato fruit is not yet known.

\section{Contribution of tomato metabolites}

to the regulation of the Salmonella yihT gene.

In an attempt to test whether metabolites or hydrophobic secondary products associated with ripening affect regulation of the yihT RIVET reporter, the effect of the hydrophobic extract of red and green tomato fruit ('Ailsa Craig') and commercially available metabolites on the reporter were tested in soft agar, as previously described (Noel et al. 2010a). Assays were conducted in M9 minimal medium an' also in $1 / 10$ diluted LB broth. In general, resolution of the reporter was lower in $1 / 10$ LB compared with M9; however, these differences are almost certainly not due to the differences in the growth rates, because the resolution of the RIVET reporter is not affected by growth rates.

Hydrophobic compounds extracted from red or green tomato fruit did not elicit significant responses in the yihT reporter, although the extracts of green (34 dpa) fruit of 'Ailsa Craig' were somewhat more stimulatory than the extracts of the red (46 dpa) fruit. However, the resolution of the reporter in response to extracts or pure compounds did not reach levels observed in green tomato fruit (Table 2 versus Figs. 2 and 3). In the presence of linoleic acid, expression of the yihT reporter was essentially eliminated (Table 2). To ascertain that linoleic acid affects the reporter rather than the activity of the TnpR resolvase, the lac $Z$ activity of the same RIVET reporter was tested and was similarly reduced (data not shown). The role of linoleic acid in the elimination of the expression of yihT is surprising, because the amounts of linoleic acid are higher in green tomato fruit (compared with red tomato fruit) and it was linoleic acid that was responsible for the upregulation of Salmonella fadH in green tomato fruit (Noel et al. 2010a).

Jasmonic acid and its biosynthetic precursors $(\gamma$-linolenic acid, 13(S)-HpOTrE( $\gamma$ ) and 12-oxo-phytodienoic acid) strongly reduced activity of the yihT reporter (Table 2). However, this response may be fairly generic because other fatty acids (decanoic and linoleic but not palmitic) affected the reporter (Table 2 ). It is tempting to speculate that, in response to the detection of the plant defense compounds such as jasmonate (and its precursors) and ethylene, Salmonella spp. modify expression of the genes involved in the synthesis of mitogen-associated molecular patterns. However, further evidence is needed in support of this hypothesis.

\section{Evidence for the involvement of a higher-level regulator in controlling Salmonella gene regulation in response to tomato ethylene signaling.}

The RIVET reporters used in this study are all in structural genes, themselves subject to control by various regulators, including AgfD (Gibson et al. 2006) and RpoS (Zaragoza et al. 2012). Because AgfD is the only identified regulator of the yih operons, we tested regulation of another AgfD-dependent gene $(a g f B)$ in 'Ailsa Craig' tomato fruit and its isogenic rin/rin and $\mathrm{Nr} / \mathrm{Nr}$ mutants to establish whether a correlation exists between expression of yihT and $a g f B$ and found a modest (but statistically significant) linear correlation between yihT and $a g f B$ expression under these conditions (Fig. 4).

These observations are intriguing because they suggest that a higher-level regulator, common to at least two genes involved in persistence of Salmonella spp. within alternate hosts, is capable of detecting a cue produced by tomato fruit that likely involves an intact ethylene synthesis or response pathway. At this time, neither the sensor nor the cue perceived by it is known.

One may hypothesize that AgfD serves as an integration point of this signaling input. AgfD is a member of the Uhp (FixJ) family of transcriptional regulators which contain a predicted receiver domain (including a conserved phosporylation site) (Romling et al. 2000). A cognate sensor kinase for AgfD is not known and, once identified, the search for the signal or an environmental condition perceived by it can proceed more efficiently. It will likely reveal novel layers of signal exchange between enteric pathogens and their alternate hosts. 


\section{MATERIALS AND METHODS}

Bacterial strains.

The construction of TIM2263 ( $\Delta y i h T 27:: k a n)$ and its derivative JTN203 $\Delta y$ ihT27-tnpR-lacZ yjeP::res1-tetRA-res1 in $S$. enterica sv. Typhimurium ATCC14028 background has been described previously (Noel et al. 2010a; Zaragoza et al. 2012). $S$. enterica sv. Typhimurium JTN202 (14028 $\Delta$ agfC37tnpRlacZ yjeP::res1-tetRA-res1) was constructed previously (Noel et al. 2010a). Because the promoterless tnpR-lac $Z$ cassette in this reporter replaces the $a g f C$ open reading frame within the operon, this reporter is considered to serve as a read-out for the regulation of the $a g f B$ promoter, which is the first gene of the operon.

The $y i h X$ RIVET reporter (CEC0023) was constructed essentially as by Osorio and associates (2005). The RIVET reporter was constructed as a merodiploid, in which the promoterless bicistronic tnpR-lac $Z$ cassette was cloned downstream from the predicted $y i h X$ promoter and the wild-type copy of the promoter was reconstituted downstream. A fragment spanning the predicted promoter region was amplified using $S$. enterica sv. Typhimurium 14028 genomic DNA as a template, with primers ctcgagCAACGAAGCCAGCGTAGTGA and ctcgagATCGGCAGCGGAAAAACCTT (introduced XhoI sites are shown in lowercase). The fragment amplified with $T a q$ polymerase (New England Biolabs, Beverly, MA, U.S.A.) was cloned into pCR2.1 and then released with XhoI. The resulting fragment was gel purified and ligated into pGOA1193 (Osorio et al. 2005) linearized with XhoI and treated with calf intestinal alkaline phosphatase. The ligation reaction was transformed into $E$. coli $\mathrm{DH} 5 \alpha$ $\lambda$ pir. The presence of the insert was verified by polymerase chain reaction (PCR) and sequencing at the University of Florida Interdisciplinary Center for Biotechnology Research Core Facility (Gainesville, FL, U.S.A.) using primer MT59, which binds within tnpR (Noel et al. 2010b). The sequenced plasmid was electroporated into E. coli BW20767 and then mated with S. enterica sv. Typhimurium JS246 (Merighi et al. 2005). Transconjugants were selected on M9 agar containing $0.4 \%$ glucose, ampicillin, tetracycline, and $\mathrm{X}$-gal at $37^{\circ} \mathrm{C}$. Individual colonies were restreaked onto fresh plates to purity. Integration of the promoter-tnpR-lac $Z$ fusion into the chromosome was confirmed by PCR using primer CTCGTCACCAGCGTAGGC (which binds upstream of the initial forward primer used to amplify the target fragment) and MT59. Once confirmed, the construct was stored in glycerol from an overnight culture in LB with ampicillin and tetracycline.

For fitness tests, a strain of serovar Typhimurium (CEC1000) marked with a kanamycin-resistance cassette in a presumed neutral site of the genome was constructed. The frt-kan-frt cassette was inserted in the intergenic region upstream of phoN (between STM4318 and STM4319, phoN) using Datsenko and Wanner (2000) mutagenesis and primers GCTTCAGCCGGAC AACGACGTCATGACGGTCTGGACGGACTGCAAACGGt gtaggctggagctgcttcg and AGAATGCCTTTGGTTTCCCCGAT TCGCATGAATTCAACGCCCCCTTCCCcatatgaatatcctccttag. The insertion was confirmed with primers GGGAGTTCGGG CAAAGCA and K2 (Datsenko and Wanner 2000).

To complement the yihT mutation in S. enterica sv. Typhimurium TIM2263, a wild-type copy of the gene with a 552-bp region upstream of yihUT, presumed to contain a native promoter, was amplified with Vent Taq-catalyzed PCR with primers ctatgtggatccACATCCCACTGCGAAAAATC and tgacta aagcttTTAGAGGCATCTACCACGCC and serovar Typhimurium 14028 genomic DNA as a template (sequences in uppercase bind to the Salmonella chromosome, BamHI and HindIII restriction sites are shown in lowercase, and anchor sequences upstream of the restriction sites are indicated by italics). The PCR product was digested with BamHI and HindIII, then

\section{Correlation between yihT and agfB expression in tomatoes}

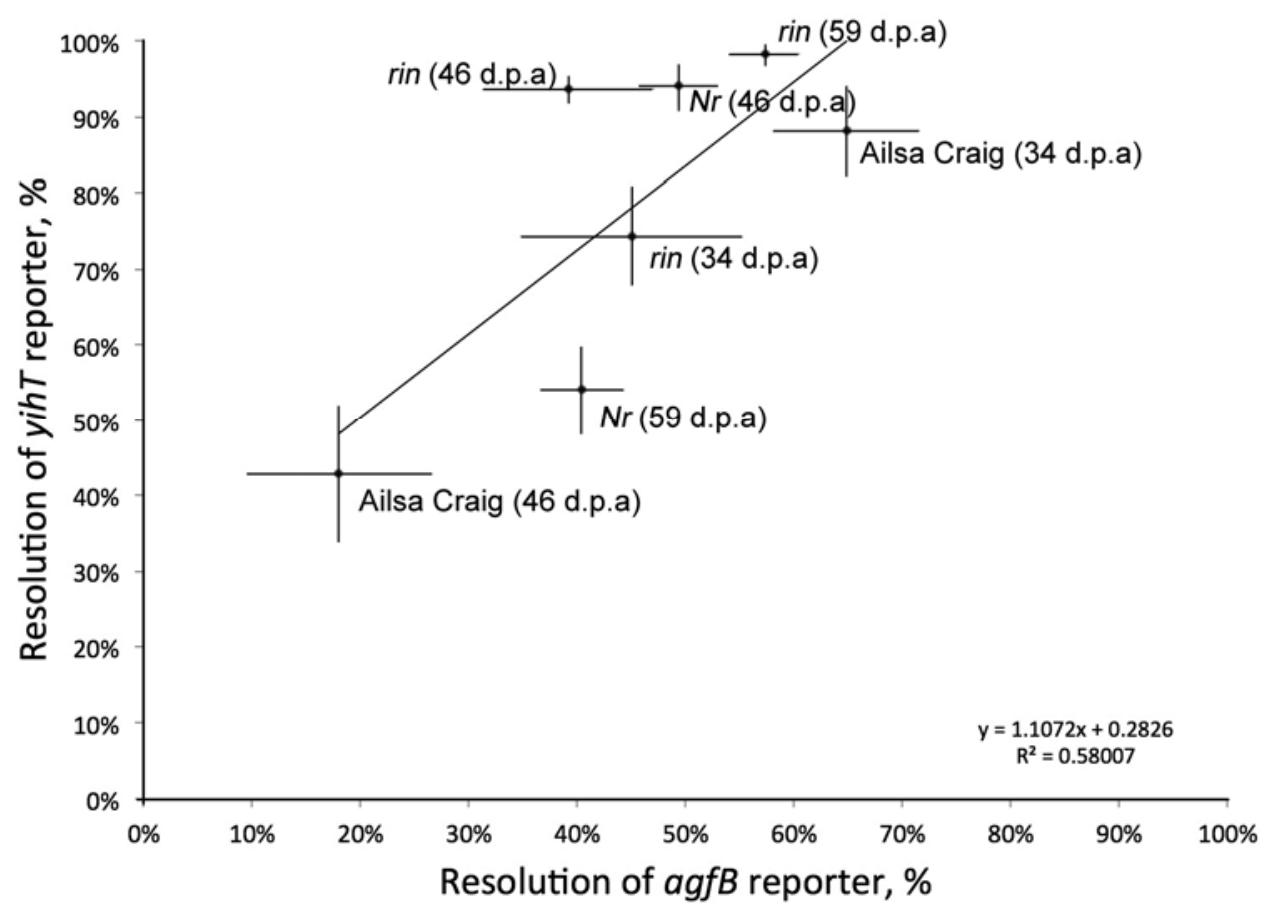

Fig. 4. Co-regulation of the AgfD-regulated recombinase-based in vivo expression technology (RIVET) reporters in tomato. To test whether the tomato environment similarly affects two RIVET reporters, each controlled by the Salmonella response regulator AgfD, resolution of JTN203 and JTN202 was tested in tomato fruit of 'Ailsa Craig' and the homozygous rin and $\mathrm{Nr}$ mutants. Tomato fruit were harvested at 34, 46, or 57 days postanthesis (d.p.a.). Each data point represents average resolution of the reporters in a specific tomato mutant or wild type. Error bars are standard deviations of three technical and three biological replications. 
cloned into pWSK29 digested with the same enzymes. The ligation reaction was transformed into chemically competent E. coli $\mathrm{DH} 5 \alpha$. The insertion was confirmed by PCR and sequencing at the DNA Lab, Arizona State University. In the resulting plasmid pMMA1, the yihUT genes with their own upstream region are cloned downstream from the lac promoter (which is not regulated in Salmonella spp. and is constitutive) and in the same orientation as the Plac. The pMMA1 plasmid was first electroporated into the restriction-minus modification-plus serovar Typhimurium JS198, from which the plasmid was extracted using the QIAprep miniprep kit (Qiagen, Hilden, Germany) according to the manufacturer's instructions and electroporated into TIM2263 ( $\Delta y$ ihT27::kan). Strains with the vector (pWSK29) only were prepared similarly to controls.

\section{Culture conditions and reporter assays.}

All strains were maintained as frozen glycerol stocks, and were subcultured into LB with appropriate antibiotics (kanamycin at $50 \mu \mathrm{g} / \mathrm{ml}$, ampicillin at $200 \mu \mathrm{g} / \mathrm{ml}$, and tetracycline at $10 \mu \mathrm{g} / \mathrm{ml})$ prior to the experiments. For plate assays, bacteria were seeded into soft LB or M9 agar $(0.3 \%$ agar) with or without X-gal $(40 \mu \mathrm{g} / \mathrm{ml})$, as indicated in the text.

For the RIVET assays in tomato, Salmonella cultures were grown at $37^{\circ} \mathrm{C}$ overnight in LB supplemented with tetracycline. Bacterial cultures were then pelleted, washed three times in an equal volume of sterile phosphate-buffered saline (PBS), and diluted to $10^{8} \mathrm{CFU} / \mathrm{ml}$. Approximately $10^{5} \mathrm{CFU}$ (in $3 \mu \mathrm{l}$ of water) were inoculated onto superficial 1-mm-deep wounds on surfaces of unwaxed fruit. At least three technical (individual infections) and two biological (tomato fruit from different plants) replications were carried out for each experiment. Unless otherwise stated, infected tomato fruit were incubated at $22^{\circ} \mathrm{C}$ in vented chambers. All RIVET assays were carried out for a week. To harvest samples, 15 -by-0.5-mm cores were removed from fruit and homogenized in PBS, and aliquots were then plated onto xylose-lysine deoxycholate (XLD) agar (Oxoid, Hampshire, U.K.) with appropriate antibiotics. Individual colonies were then patched on LB agar with tetracycline to detect constructs in which TnpR recombinase was active.

\section{Plant materials.}

All assays were initially conducted in unwaxed red ripe Campari tomato fruit purchased at local supermarkets. Followup experiments, as indicated in text, were carried out with mature green or fully ripe tomato fruit of 'Amish Salad', 'Bonny Bes't, 'Celebrity', 'Red Calabash', 'Sebring', 'Solar Fire', 'Mariana', 'Bloody Butcher', 'Kumato', and 'Snow White'. Note that, at maturity (corresponding to the United States Department of Agriculture stages 5 and 6), fruit of 'Amish Salad', 'Bonny Best', 'Celebrity', 'Red Calabash', 'Sebring', 'Solar Fire', 'Mariana', and 'Bloody Butcher' turn red; 'Kumato' are brown; and 'Snow White' are ivory. Other than these pigmentation differences, 'Kumato' and 'Snow White' ripen normally. The brown color of ripe tomato fruit of 'Kumato' is due to an incomplete turnover of chlorophyll in mature tomato fruit. Seed was purchased from commercial suppliers. Tomato plants were grown in the field (two locations and seasons: Citra, FL in fall 2010 [conventional] and Archer, FL in spring 2012 [transitional organic]) or in the roof-top greenhouse (during the breaks between production seasons). For each variety, field and greenhouse-grown tomato fruit were sampled and the combined data are presented.

'Ailsa Craig' and lines nearly isogenic for the rin and $\mathrm{Nr}$ mutations (Vrebalov et al. 2002; Yen et al. 1995) were grown in the roof-top greenhouse in spring and summer 2012. To track developmental stages of the fruit, each developing fruit was tagged when it first reached exactly $1 \mathrm{~cm}$ in diameter, equal to 7 dpa (Alba et al. 2005). In the greenhouse, plants were grown from seed in Miracle-Gro potting soil and fertilized biweekly with Miracle-Gro tomato plant food (18-21-21) (Marysville, OH, U.S.A.).

For all samplings, fruit were harvested individually from the field- and greenhouse-grown plants, ensuring that ripe and unripe fruit were from the same plants to reduce any variability associated with differences in the associated endo- or epimicrobiota. All tomato fruit were inoculated with Salmonella spp. within 3 to $4 \mathrm{~h}$ of harvest.

For genotyping experiments, plants were grown in the greenhouse from seed to the approximately four- to six-true-leaf stage. DNA was extracted with a PowerPlant DNA isolation kit (MoBio, Carlsbad, CA, U.S.A.) according to the manufacturer's instructions. Genotyping for Rin/rin alleles was conducted by PCR using primers ATACGATAATGTACAACCCGAAAATG and TCAACTTGAACACACATAAAAAGGAA, yielding a 330-bp fragment diagnostic of the wild-type Rin allele, and primers CTTTCAAACATCATGGCATTGTGGTG and ATAT CATTGGCGGAACTTGACGTGAG, yielding a 765-bp fragment diagnostic for the mutant rin allele.

\section{Fitness of the Salmonella mutants.}

To calculate a competitive index, wild-type S. enterica sv. Typhimurium 14028 and isogenic mutants were seeded at $10^{5}$ CFU/infection, roughly at a 1:1 ratio into tomato. In parallel, S. enterica sv. Typhimurium 14028 and its isogenic kanamycin-resistant strain CEC1000 were similarly inoculated onto eight tomato fruit, three wounds per fruit. All samples were incubated for a week at $22^{\circ} \mathrm{C}$ in vented chambers (with the exception of the ethylene add-back experiments; discussed below). Upon completion of the incubation, tomato fruit were inspected visually and those with signs of spoilage were discarded. At harvest, tomato fruit were macerated in a stomacher with an equal volume of PBS, then dilution plated onto XLD plates, which were incubated at $42^{\circ} \mathrm{C}$. The relative ratios of the strains in the inocula and in the recovered samples were calculated by dilution plating and patching on antibiotic containing media. Competitive indices were calculated for each treatment using the formula $\left(\mathrm{M}_{\text {out }} / \mathrm{WT}_{\text {out }}\right) /\left(\mathrm{M}_{\text {in }} / \mathrm{WT}_{\text {in }}\right)$, where $\mathrm{M}$ is the proportion of mutant cells and WT is the proportion of the wild-type cells in the inocula (in) or in the recovered samples (out). Log-transformed values of competitive index are presented. The statistical and biological significance of each competitive index was established by comparing log values of the competitive indices of each pair to the log of competitive index similarly calculated for ATCC 14028 versus CEC1000, using the analysis of variance test $(P<0.05)$. It is important to recognize that, because of the way the fitness of the mutant is tested within the co-infection with the wild type, an increase in the fitness of the mutant could also be a consequence of the reduced fitness of the wild type under some conditions.

Extraction of hydrophobic components from tomato fruit.

Mature green ( $34 \mathrm{dpa}$ ) or red ripe (46 dpa) tomato fruit of 'Ailsa Craig' were blended and mixed with two volumes of chloroform. The organic phase was filtered (Whatman filter paper), rotary evaporated, and freeze dried overnight. For bioassays, $5 \mathrm{mg}$ of lyophilized compounds were resuspended in $40 \mu \mathrm{l}$ of dimethyl sulfoxide (DMSO). Controls containing only DMSO and distilled water were also tested. A volume of the extract corresponding to approximately 0.4 tomato "equivalents" was added to the LB $0.3 \%$ agar plates for the bioassays.

\section{Ethylene add-back experiments.}

Tagged, developmentally synchronized tomato fruit ("Ailsa Craig' wild type and $\mathrm{rin} / \mathrm{rin}$ ) were harvested at 34 and 46 dpa. 
Tomato fruit were inoculated with an equal mix of TIM2263 (AyihT27::kan) and serovar Typhimurium 14028 exactly as above for fitness tests. Infected tomato fruit were placed inside a 40-by-40-by-20-cm lidded, air-tight aquarium, into which $0.39 \mathrm{ml}$ of $100 \%$ ethylene was injected with a syringe for a treatment concentration of $12 \mathrm{ppm}$. Tomato fruit were incubated for 1 week and ethylene injections were repeated every $48 \mathrm{~h}$, following a brief (approximately $10 \mathrm{~min}$ ) venting to reduce accumulation of $\mathrm{CO}_{2}$. Upon completion of the experiment, the 'Ailsa Craig' tomato fruit turned red while the rin/rin remained green. Tomato fruit were harvested and the competitive index was calculated as described above.

\section{ACKNOWLEDGMENTS}

This research was supported by the United States Department of Agriculture (USDA)-NRI AFRI grant 2011-67017-30127 (to M. Teplitski and J. J. Giovannoni). Construction of the phoN mutant was funded as a part of the project supported by the Center for Produce Safety, University of California-Davis (agreement number 201300842-01) and the California Department of Food and Agriculture's Specialty Crop Block Grant Program (SCB12064). C. E. Cox gratefully acknowledges USDA NIFA postdoctoral fellowship CRIS 0229689. We thank M. Moraes for assistance with designing primers to construct CEC1000.

\section{LITERATURE CITED}

Alba, R., Payton, P., Fei, Z., McQuinn, R., Debbie, P., Martin, G. B., Tanksley, S. D., and Giovannoni, J. J. 2005. Transcriptome and selected metabolite analyses reveal multiple points of ethylene control during tomato fruit development. Plant Cell 17:2954-2965.

Angelichio, M.J., and Camilli, A. 2002. In vivo expression technology. Infect. Immun. 70:6518-6523.

Arrach, N., Zhao, M., Porwollik, S., Hoffman, R. M., and McClelland, M. 2008. Salmonella promoters preferentially activated inside tumors. Cancer Res. 68:4827-4832.

Arrach, N., Cheng, P., Zhao, M., Santiviago, C.A., Hoffman, R. M., and McClelland, M. 2010. High-throughput screening for salmonella avirulent mutants that retain targeting of solid tumors. Cancer Res. 70:21652170 .

Barak, J. D., and Schroeder, B. K. 2012. Interrelationships of food safety and plant pathology: The life cycle of human pathogens on plants. Annu. Rev. Phytopathol. 50:241-266.

Barak, J. D., Jahn, C. E., Gibson, D. L., and Charkowski, A. O. 2007. The role of cellulose and $\mathrm{O}$-antigen capsule in the colonization of plants by Salmonella enterica. Mol. Plant-Microbe Interact. 20:1083-1091.

Barak, J. D., Kramer, L. C., and Hao, L. Y. 2011. Colonization of tomato plants by Salmonella enterica is cultivar dependent, and type 1 trichomes are preferred colonization sites. Appl. Environ. Microbiol. 77:498-504.

Barry, C. S., McQuinn, R. P., Chung, M. Y., Besuden, A., and Giovannoni, J. J. 2008. Amino acid substitutions in homologs of the STAY-GREEN protein are responsible for the green-flesh and chlorophyll retainer mutations of tomato and pepper. Plant Physiol. 147:179-187.

Batz, M. B., Hoffman, S., and Morris, J. G. 2011. Ranking the Risks: The 10 Pathogen-Food Combinations with the Greatest Burden on Public Health. University of Florida, Emerging Pathogens Institute, Gainesville, FL, U.S.A.

Brandl, M. T., Cox, C. E, and Teplitski, M. 2013. Salmonella interactions with plants and their associated microbiota. Phytopathology 103:316-325

Carrari, F., Baxter, C., Usadel, B., Urbanczyk-Wochniak, E., Zanor, M. I., Nunes-Nesi, A., Nikiforova, V., Centero, D., Ratzka, A., Pauly, M., Sweetlove, L. J., and Fernie, A. R. 2006. Integrated analysis of metabolite and transcript levels reveals the metabolic shifts that underlie tomato fruit development and highlight regulatory aspects of metabolic network behavior. Plant Physiol. 142:1380-1396.

Danyluk, M. D., Nozawa-Inoue, M., Hristova, K. R., Scow, K. M. Lampinen, B., and Harris, L. J. 2008. Survival and growth of Salmonella Enteritidis PT 30 in almond orchard soils. J. Appl. Microbiol. 104:1391-1399.

Datsenko, K. A., and Wanner, B. L. 2000. One-step inactivation of chromosomal genes in Escherichia coli K-12 using PCR products. Proc. Natl. Acad. Sci. U.S.A. 97:6640-6645.

Ge, S. C., Danino, V., He, Q. S., Hinton, J. C. D., and Granfors, K. 2010. Microarray analysis of response of Salmonella during infection of
HLA-B27-transfected human macrophage-like U937 cells. BMC Genomics 11:456. Published online.

Gibson, D. L., White, A. P., Snyder, S. D., Martin, S., Heiss, C., Azadi, P. Surette, M., and Kay, W. W. 2006. Salmonella produces an O-antigen capsule regulated by AgfD and important for environmental persistence. J. Bacteriol. 188:7722-7730.

Greene, S. K., Daly, E. R., Talbot, E. A., Demma, L. J., Holzbauer, S., Patel, N. J., Hill, T. A., Walderhaug, M. O., Hoekstra, R. M., Lynch, M. F., and Painter, J. A. 2008. Recurrent multistate outbreak of Salmonella Newport associated with tomatoes from contaminated fields, 2005. Epidemiol. Infect. 136:157-165.

Mandrell, R. 2009. Enteric human pathogens associated with fresh produce: Sources, transport, and ecology. In: Microbial Safety of Fresh Produce. X. Fan, B. A. Niemira, C. J. Doona, F. E. Feeherry, and R. B. Gravani, eds. Blackwell Publishing and the Institute of Food Technologies, Ames, IA, U.S.A.

Martel, C., Vrebalov, J., Tafelmeyer, P., and Giovannoni, J.J. 2011. The tomato MADS-box transcription factor RIPENING INHIBITOR interacts with promoters involved in numerous ripening processes in a COLORLESS NONRIPENING-dependent manner. Plant Physiol. 157:15681579.

Merighi, M., Ellermeier, C. D., Slauch, J. M., and Gunn, J. S. 2005. Resolvase-in vivo expression technology analysis of the Salmonella enterica serovar Typhimurium PhoP and PmrA regulons in BALB/c mice. J. Bacteriol. 187:7407-7416.

Morgan, E., Campbell, J. D., Rowe, S. C., Bispham, J., Stevens, M. P., Bowen, A. J., Barrow, P. A., Maskell, D. J., and Wallis, T. S. 2004. Identification of host-specific colonization factors of Salmonella enterica serovar Typhimurium. Mol. Microbiol. 54:994-1010.

Noel, J. T., Arrach, N., Alagely, A., McClelland, M., and Teplitski, M. 2010a. Specific responses of Salmonella enterica to tomato varieties and fruit ripeness identified by In vivo expression technology. PLoS One 5. Published online.

Noel, J. T., Joy, J., Smith, J. N., Fatica, M., Schneider, K. R., Ahmer, B. M., and Teplitski, M. 2010b. Salmonella SdiA recognizes N-acyl homoserine lactone signals from Pectobacterium carotovorum in vitro, but not in a bacterial soft rot. Mol. Plant-Microbe Interact. 23:273-282.

Osorio, C. G., Crawford, J. A., Michalski, J., Martinez-Wilson, H., Kaper, J. B., and Camilli, A. 2005. Second-generation recombination-based in vivo expression technology for large-scale screening for Vibrio cholerae genes induced during infection of the mouse small intestine. Infect. Immun. 73:972-980.

Parker, C. T., Kyle, J. L., Huynh, S., Carter, M. Q., Brandl, M. T., Mandrell, R. E. 2012. Distinct transcriptional profiles and phenotypes exhibited by Escherichia coli O157:H7 isolates related to the 2006 spinachassociated outbreak. Appl. Environ. Microbiol. 78:455-463

Romling, U., Rohde, M., Olsen, A., Normark, S., and Reinkoster, J. 2000. AgfD, the checkpoint of multicellular and aggregative behaviour in Salmonella Typhimurium regulates at least two independent pathways. Mol. Microbiol. 36:10-23.

Santiviago, C. A., Reynolds, M. M., Porwollik, S., Choi, S. H., Long, F., Andrews-Polymenis, H. L., and McClelland, M. 2009. Analysis of pools of targeted Salmonella deletion mutants identifies novel genes affecting fitness during competitive infection in mice. PLoS Pathog. 5:e1000477. Published online.

Shi, X., Namvar, A., Kostrzynska, M., Hora, R., and Warriner, K. 2007. Persistence and growth of different Salmonella serovars on pre- and postharvest tomatoes. J. Food Prot. 70:2725-2731.

Teplitski, M., Noel, J. T., Alagely, A., and Danyluk, M. D. 2012. Functional genomics studies shed light on the nutrition and gene expression of non-typhoidal Salmonella and enterovirulent $E$. coli in produce. Food Res. Int. 45:576-586.

Tyler, H. L., and Triplett, E. W. 2008. Plants as a habitat for beneficial and/or human pathogenic bacteria. Annu. Rev. Phytopathol. 46:53-73.

Uesugi, A. R., Danyluk, M. D., Mandrell, R. E., and Harris, L. J. 2007. Isolation of Salmonella Enteritidis phage type 30 from a single almond orchard over a 5-year period. J. Food Prot. 70:1784-1789.

Vrebalov, J., Ruezinsky, D., Padmanabhan, V., White, R., Medrano, D., Drake, R., Schuch, W., and Giovannoni, J. 2002. A MADS-box gene necessary for fruit ripening at the tomato ripening-inhibitor (Rin) locus. Science 296:343-346.

Yen, H. C., Lee, S. Y., Tanksley, S. D., Lanahan, M. B., Klee, H. J., and Giovannoni, J. J. 1995. The tomato Never-Ripe locus regulates ethylene-inducible gene expression and is linked to a homolog of the Arabidopsis Etr1 gene. Plant Physiol. 107:1343-1353.

Zaragoza, W. J., Noel, J. T., and Teplitski, M. 2012. Spontaneous non-rdar mutations increase fitness of Salmonella in plants. Environ. Microbiol. Rep. 4:453-458. 